\title{
Floral trait differentiation in Anacamptis coriophora: Phenotypic selection on scents, but not on colour
}

\author{
Nina Joffard ${ }^{1,2}$ (D) | Iris Le Roncé ${ }^{1,3}$ | Alban Langlois ${ }^{1,4}$ | Julien Renoult ${ }^{1}$ | \\ Bruno Buatois $^{1}$ | Laurent Dormont ${ }^{1}$ | Bertrand Schatz ${ }^{1}$
}

${ }^{1}$ Centre d'Ecologie Fonctionnelle et Evolutive, EPHE-PSL, CNRS, Univ.

Montpellier, Univ. Paul Valéry Montpellier 3, IRD, Montpellier, France

${ }^{2}$ Evolutionsbiologiskt Centrum (EBC), Uppsala, Sweden

${ }^{3}$ Département de biologie, École Normale Supérieure de Lyon, Lyon, France

${ }^{4}$ Université Toulouse III Paul Sabatier, Toulouse, France

Correspondence

Nina Joffard, Evolutionsbiologiskt Centrum (EBC), Norbyvägen 18 D, 75236 Uppsala, Sweden.

Email: nina_joffard@hotmail.fr

\section{Funding information}

Agence Nationale de la Recherche, Grant/ Award Number: ANR-14-CE02-0012; Hautes Ecoles Sorbonne Arts et Métiers (HESAM) Université; Centre National de la Recherche Scientifique

\begin{abstract}
Current divergent selection may promote floral trait differentiation among conspecific populations in flowering plants. However, whether this applies to complex traits such as colour or scents has been little studied, even though these traits often vary within species. In this study, we compared floral colour and odour as well as selective pressures imposed upon these traits among seven populations belonging to three subspecies of the widespread, generalist orchid Anacamptis coriophora. Colour was characterized using calibrated photographs, and scents were sampled using dynamic headspace extraction and analysed using gas chromatography-mass spectrometry. We then quantified phenotypic selection exerted on these traits by regressing fruit set values on floral trait values. We showed that the three studied subspecies were characterized by different floral colour and odour, with one of the two predominant floral volatiles emitted by each subspecies being taxon-specific. Plant size was positively correlated with fruit set in most populations, whereas we found no apparent link between floral colour and female reproductive success. We detected positive selection on several taxon-specific compounds in A. coriophora subsp. fragrans, whereas no selection was found on floral volatiles of $A$. coriophora subsp. coriophora and $A$. coriophora subsp. martrinii. This study is one of the first to document variation in phenotypic selection exerted on floral scents among conspecific populations. Our results suggest that selection could contribute to ongoing chemical divergence among A. coriophora subspecies.
\end{abstract}

KEYWORDS

Anacamptis coriophora, floral colour, floral scents, phenotypic selection, pollination

\section{INTRODUCTION}

One of the most striking characteristics of Angiosperms is their floral diversity, which has been the focus of a fruitful research for over two centuries (Darwin, 1862; Harder \& Barrett, 2006; Sprengel,
1793). Because floral traits act as visual, olfactory or even tactile signals maximizing attractiveness to pollinators and pollen transfer efficiency (Burger, Dötterl, \& Ayasse, 2010; Jersáková, Jürgens, Šmilauer, \& Johnson, 2012; Schiestl \& Johnson, 2013), they have a major influence on plant reproductive success. It is thus generally 
assumed that floral evolution has been driven by pollinator-mediated selection (Harder \& Johnson, 2009; Sletvold, Grindeland, \& Ågren, 2010), although antagonists (Irwin, Bronstein, Manson, \& Richardson, 2010; McCall, Murphy, Venner, \& Brown, 2013; Parachnowitsch \& Caruso, 2008; Ramos \& Schiestl, 2019), co-occurring plants (Caruso, 2000, 2001) and abiotic factors (Arista, Talavera, Berjano, \& Ortiz, 2013; Armbruster, 2002) may also impose direct or indirect selective pressures upon floral characteristics (Caruso, Eisen, Martin, \& Sletvold, 2019; Strauss \& Whittall, 2006).

Floral traits often vary among conspecific populations (Anderson, Ros, Wiese, \& Ellis, 2014; Breitkopf et al., 2013; Dormont, Joffard, \& Schatz, 2019; Ellis \& Johnson, 2009). If such variation is adaptive, we may detect current divergent selection on floral traits when comparing these populations (i.e., linear selection in opposite directions, stabilizing selection with different optima, or, for multivariate traits, selection acting on distinct components of the trait). This was shown to be the case for diverse floral characteristics, including phenology (Hall \& Willis, 2006; Sandring, Riihimäki, Savolainen, \& Ågren, 2007), size (Chapurlat, Ågren, \& Sletvold, 2015; Totland, 2001) and shape (Gómez et al., 2008; Gómez, Perfectti, Bosch, \& Camacho, 2009; Johnson \& Steiner, 1997; Soteras, Rubini Pisano, Bariles, Moré, \& Cocucci, 2020). In the generalist plant Erysimum mediohispanicum, for example, phenotypic selection on corolla shape was found to vary among populations visited by distinct pollinators and to match the observed pattern of morphological differentiation (Gómez et al., 2008, 2009), suggesting that current divergent selection promotes ongoing floral trait differentiation in this species.

Among floral traits, colour and scents are of particular importance for biotic interactions, especially for pollinator attraction (Raguso, 2008; Whitehead \& Peakall, 2009). Visual and olfactory floral signals have been shown to vary among conspecific populations in many plant species (reviewed in Delle-Vedove, Schatz, \& Dufay, 2017; Dormont et al., 2019). Yet, surprisingly little is known regarding the processes promoting such variation, although adaptation to divergent pollination niches is often proposed as the most likely explanation (Breitkopf et al., 2013; Chess, Raguso, \& LeBuhn, 2008). In particular, we are aware of only one study that has explicitly linked variation in floral colour and scents among conspecific populations to variation in phenotypic selection exerted on these traits (Gross, Sun, \& Schiestl, 2016).

In this study, we ask whether current divergent selection could contribute to ongoing floral colour and scent differentiation in the widespread, generalist orchid species Anacamptis coriophora. This species comprises several subspecies, three of which are known to occur in France, namely Anacamptis coriophora subsp. coriophora (hereafter referred to as 'A. c. coriophora'), A. coriphora subsp. fragrans (hereafter referred to as 'A. $c$. fragrans') and A. coriophora subsp. martrinii (hereafter referred to as 'A. c. martrinii'). These three subspecies grow in different habitats and are described as having distinct floral colour and scents (Bournérias \& Prat, 2005; Delforge, 2006), although this has never been properly quantified. Specifically, flowers of $A$. c. fragrans are described as having a lighter colour than those of A. c. coriophora and A. c. martrinii, and a sweeter smell than those of A. c. coriophora, whereas flowers of A. c. martrinii are described as odourless (Delforge, 2006). By characterizing floral colour and scents in seven populations of this species, we first aim at describing variation in floral phenotypes among and within subspecies. We then ask whether selection could explain such variation by measuring phenotypic selection gradients for floral colour and scent traits in each population, and by comparing selective pressures imposed upon these traits among and within subspecies. In particular, if floral trait differentiation between the three studied subspecies is adaptive, we expect selection to favour plants with dark flowers in A. c. coriophora and A. c. martrinii but plants with light flowers in A. c. fragrans. Our predictions regarding selection exerted on floral scents depend on the observed pattern of chemical differentiation. If the three subspecies differ in terms of total emission rate (e.g., if A. c. martrinii truly is odourless), we may detect current divergent selection on this trait; but if they differ in terms of scent chemistry (e.g., if A. c. coriophora and A. c. fragrans truly have distinct smells), we expect selection to favour plants emitting high amounts of taxon-specific compounds, and/or to vary in direction or strength when acting on the same compounds.

\section{2 | MATERIALS AND METHODS}

\section{1 | Studied taxa and populations}

Anacamptis coriophora (L.) Bateman, Pridgeon \& Chase (1997) is a terrestrial, widespread orchid species which is distributed across central and southern Europe. This species produces nectar in a short spur (i.e., unlike other Anacamptis species, it is 'rewarding' and not 'deceptive') and is pollinated by various bee species (Claessens \& Kleynen, 2011; Dafni \& Ivri, 1979; Joffard, Massol, Grenié, Montgelard, \& Schatz, 2019). It comprises several subspecies, three of which are known to occur in France, namely Anacamptis $c$. coriophora, A. c. fragrans and A. c. martrinii (Figure 1a). Anacamptis c. coriophora grows in low to medium altitude hay meadows and seasonally wet to wet grasslands, whereas A. c. fragrans grows in dry Mediterranean grasslands and A. c. martrinii in mountain hay meadows from the Pyrenees (Bournérias \& Prat, 2005; Delforge, 2006). These three subspecies display inflorescences with about 10-30 flowers that open in acropetal sequence (from basal to distal) between May and July. Their flowers are light (in A. c. fragrans) to dark (in A. c. coriophora and A. c. martrinii) pink with red spots and sometimes green highlights on the labellum, and they are odorous, $A$. c. coriophora flowers having a stinkbug-like smell and A. c. fragrans flowers a sweeter, vanilla-like smell (Bournérias \& Prat, 2005; Delforge, 2006). Note that A. c. coriophora and A. c. fragrans are sometimes treated as bona fide species (e.g., see Bateman et al., 2003); however, their presumed phylogenetic distinctness has been established using a single accession per taxon and a single locus. Because we think that more data are needed to conclusively demonstrate that these two taxa truly represent different species, in this study we adopt the more conservative view that they represent subspecies of $A$. coriophora. 


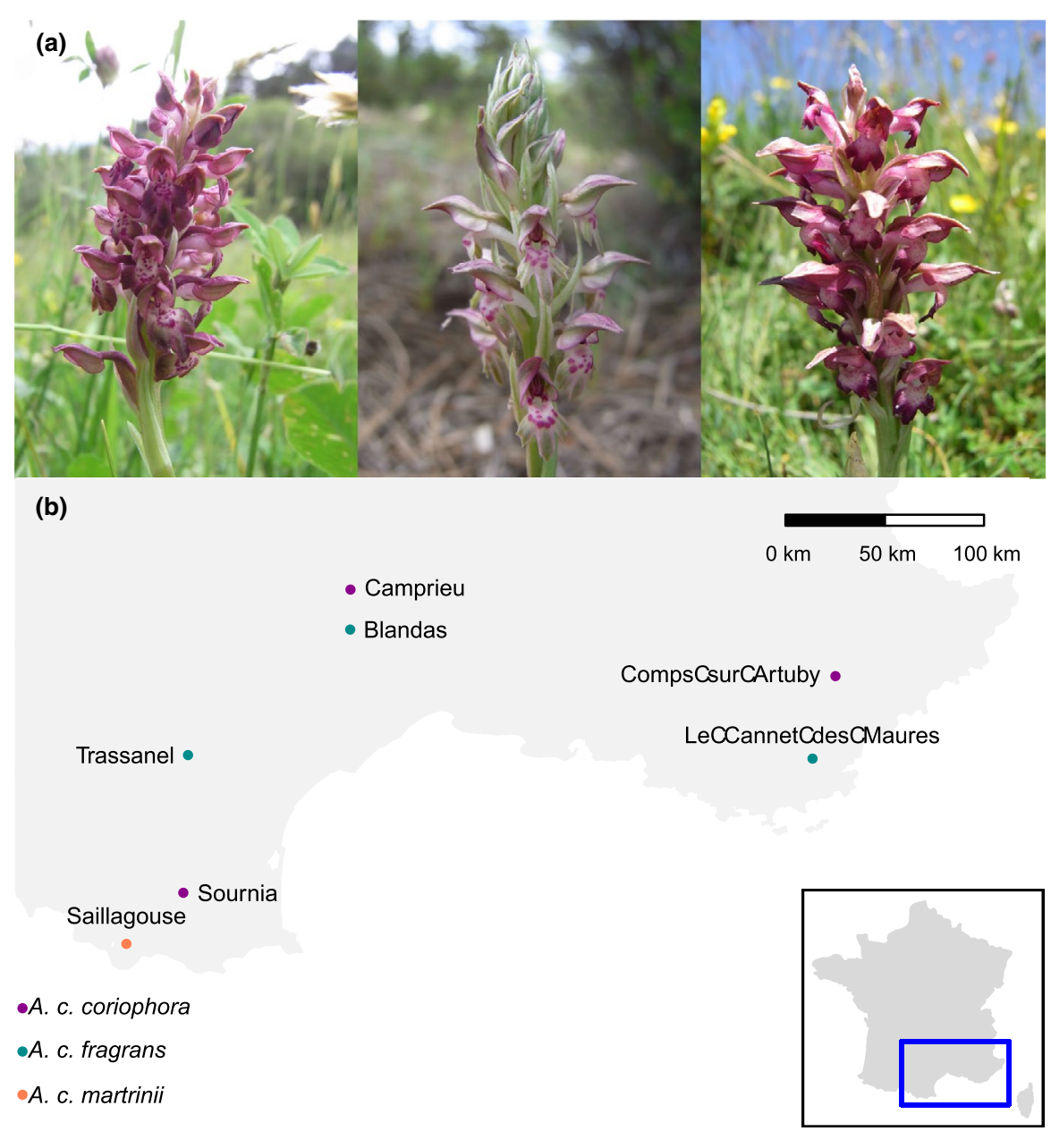

FIGURE 1 (a) Photographs of the inflorescences of Anacamptis coriophora subsp. coriophora, A. coriphora subsp. fragrans and A. coriophora subsp. martrinii (from left to right). (b) Geographic distribution of populations of $A$. coriophora subsp. coriophora (in purple), A. coriphora subsp. fragrans (in turquoise) and $A$. coriophora subsp. martrinii (in orange) sampled in this study

Three populations of A. c. coriophora, three populations of A. c. fragrans and one population of $A$. c. martrinii-each comprising between 50 and 100 individuals-were sampled in southern France during spring/summer 2016 (Figure 1b). Populations were assigned to subspecies using morphology, phenology and habitat characteristics. These populations were selected in order to sample both A. c. coriophora and A. c. fragrans in three geographical regions (south-eastern, south-central and south-western France). Repeated sampling of two of these seven populations (one of A. c. coriophora and one of A. c. fragrans) was planned in 2017 in order to test whether phenotypic selection patterns were consistent across years, but those were almost entirely destroyed due to bad weather conditions.

\subsection{Fieldwork}

\subsection{1 | Measurement of floral traits}

In each of the seven populations, 50-65 individuals were randomly selected and marked. For each individual, plant height and inflorescence length (distance between uppermost and lowermost flowers) were measured to the nearest $0.5 \mathrm{~mm}$ with a tape measure and flowers were counted. Plant height, inflorescence length and number of flowers were used as proxies of plant size.
In six populations of seven (three of $A$. c. coriophora, two of $A$. c. fragrans and one of $A$. c. martrinii), floral colour was characterized using calibrated photographs. This method was chosen over spectrophotometry because it allows characterizing the colour of the entire labellum whereas spectrophotometry focuses on one or a few subjectively selected points. This is especially important here since as mentioned above, A. coriophora has a nonuniform floral colour with red spots and green highlights on the labellum. For each individual, one of the lowermost flowers was photographed using a standardized procedure (front view and planar position, indirect natural sunlight) with a Nikon D70 digital camera equipped with a Novoflex $35 \mathrm{~mm}$ lens. Note that floral colour variation observed within individuals was found to be negligible compared to that observed among individuals (data not shown). A ruler and two reference greys ( $23 \%$ and $35 \%$ of average reflectance) were included in each photograph for further scaling and colour calibration, respectively. Photographs were taken in the human-visible light range (i.e., 400-700 nm), but to confirm that our results were interpretable ecologically, we produced multispectral images of ten flowers in the UV-visible light range (340-700 nm) using a UV-sensitive camera (see Method S1). These images revealed that A. coriophora flowers reflect almost no light in the UV light range (see Figure S1).

In each of the seven populations, floral odour was sampled in situ using headspace dynamic extraction, a nondestructive method 
allowing the extraction of scents emitted by the entire inflorescence. Floral odour was extracted between 08:00 hr and 18:00 hr, but daily variation in floral scent emissions was found to be negligible within the A. coriophora group (data not shown). For each individual, the inflorescence was enclosed in a 10*5 cm Nalophan ${ }^{\circledR}$ bag for $30 \mathrm{~min}$. ChromatoProbe ${ }^{\circledR}$ quartz microvials (Varian, Inc.; length: $15 \mathrm{~mm}$, inner diameter: $2 \mathrm{~mm}$ ) filled with $3 \mathrm{mg}$ of a 1:1 mix of Tenax-TA and Carbotrap $^{\circledR}$ (respectively, 60-80 and 20-40 mesh, Sigma-Aldrich) were used as adsorbent traps, and $1 \mu$ of internal standard (n-tetradecane, $100 \mathrm{ng} / \mu \mathrm{l}$ ) was injected in these traps. Two traps-one for mass spectrometry analyses, used for compound identification, and one for flame ionization detection analyses, used for compound quantification-connected to a pump (N86KNDC, KNF) by silicon tubes were inserted into the bag after $20 \mathrm{~min}$. The air inside the bag was then pumped through the two traps for $10 \mathrm{~min}$ at a rate of $40 \mathrm{ml} / \mathrm{min}$. After floral odour extraction, each trap was put into a vial and stored at $-20^{\circ} \mathrm{C}$ until further analyses. In each population, the air contained in one empty bag was sampled as a control.

In parallel, pollinator guilds were characterized in each of the seven populations and pollen limitation of female reproductive success was quantified using hand pollinations in four populations of seven (see Methods S2 and S3).

\subsection{2 | Estimation of female reproductive success}

One month after the measurement of floral traits, the fruit set of each individual was measured and used as a proxy of female reproductive success. Relative female reproductive success was calculated by dividing the number of fruits produced by each individual by the mean number of fruits produced per plant in each population, following the recommendations of Lande and Arnold (1983). Number of seeds per fruit could not be counted, as fruit collection is not allowed in A. c. coriophora, A. c. fragrans and A. c. martrinii, which are nationally protected, but fruit set varied extensively among individuals in most populations whereas fruit size (which was shown to be a good proxy of seed set in other orchid species, e.g. see Chapurlat et al., 2015) was apparently more constant. Male reproductive success could not be measured either, as it would have required recording pollinia removal regularly during the entire flowering season. In some populations, a few individuals were lost due to mowing or grazing, but at least $96 \%$ of them were retrieved and could be included in further analyses (except in Blandas, where 17\% of them were lost).

\section{3 | Floral colour analyses}

Although digital photographs can be powerful tools for measuring colour, usually pixel values cannot be compared among studies because of the model-specific nonlinear response of the camera to light intensity and because of variation in the illuminant spectrum. Moreover, even within studies pixel values may not be comparable among photographs if lighting conditions vary, which was the case here. To circumvent these limitations, each photograph was calibrated using the Image Calibration and Analysis Toolbox plugin (Troscianko \& Stevens, 2015) in the software ImageJ (Schneider, Rasband, \& Eliceiri, 2012). After this calibration step, on each photograph, the labellum was manually selected and its colour characterized using (a) RGB values, to provide a measure of floral colour as we humans perceive it, and (b) the excitation values of the SW (short wavelength) and MW (medium wavelength) photoreceptors of the honeybee (Apis mellifera; see Method S4). Including both human and insect photoreceptors in our analysis ensures that our results do not critically depend on the specificities of one visual system and therefore likely apply to other pollinator species for which photoreceptor sensitivities are unknown. For each labellum, we measured one mean and one standard deviation values for each of the R, G, B, SW and MW channels.

\section{4 | Floral odour analyses}

Floral odour was analysed by gas chromatography (GC)-mass spectrometry (MS) and flame ionization detection (FID) using a Trace 1310 gas chromatograph coupled with a flame ionization detector and an ISQ mass spectrometer (Thermo-Electron) and equipped with an OPTIMA $^{\circledR} 5$-MS-HT capillary column $(30 \mathrm{~m} \times 0.25 \mathrm{~mm} \times 0.25 \mu \mathrm{m}$, Macherey-Nagel). Traps were handled with a Multi-Purpose Sampler (Gerstel) and desorbed with a double-stage desorption system composed of a Thermal Desorption Unity (TDU) and a Cold Injection System (CIS; Gerstel). First, traps were desorbed splitless with a temperature of $250^{\circ} \mathrm{C}$ on the $\mathrm{CIS}$ trap cooled at $-80^{\circ} \mathrm{C}$ using liquid nitrogen. Then, the $\mathrm{CIS}$ trap was heated to $250^{\circ} \mathrm{C}$ with a $1: 4$ split ratio to inject the compounds into the column. Oven temperature was held at $40^{\circ} \mathrm{C}$ for $3 \mathrm{~min}$, increased from $40^{\circ} \mathrm{C}$ to $210^{\circ} \mathrm{C}$ at a rate of $5^{\circ} \mathrm{C} / \mathrm{min}$ and from 220 to $250^{\circ} \mathrm{C}$ at a rate of $10^{\circ} \mathrm{C} / \mathrm{min}$, and held at $250^{\circ} \mathrm{C}$ for $2 \mathrm{~min}$. The temperatures of transfer line and ion source of the mass spectrometer were $250^{\circ} \mathrm{C}$ and $200^{\circ} \mathrm{C}$, respectively, and acquisition was set from 38 to $350 \mathrm{~m} / \mathrm{z}$, at a $70 \mathrm{eV}$ ionization energy. The FID was heated to $250^{\circ} \mathrm{C}$. Retention times of a series of n-alkanes (Alkanes standard solution, 04070, Sigma-Aldrich ${ }^{\circledR}$ ) were used to convert retention times into retention indices. Compounds were identified on the GC-MS samples based on their retention indices and mass spectra, which were compared to those recorded in databases (Adams, 2007). Peak areas were measured on the GC-FID samples using the software Xcalibur (Thermo-Electron) and were converted to absolute (i.e., in ng) and relative (i.e., in \%) amounts using the peak area of the internal standard ( $n$-tetradecane).

\section{5 | Statistical analyses}

\subsection{1 | Variation in floral traits and female reproductive success}

Variation in plant size among populations of the same subspecies (A. c. coriophora or A. c. fragrans) was investigated using linear models 
with population as a fixed effect and plant size traits (plant height, inflorescence length or number of flowers) as response variables. Variation among subspecies was then investigated using linear mixed effects models with subspecies as a fixed effect, population as a random effect and plant size traits as response variables. Variation in floral colour (characterized by mean and $S D$ values in the $R, G, B$, MW and SW channels) and scents (characterized by log-transformed absolute amounts of each compound) among subspecies and populations of the same subspecies was investigated using Partial Least Square-Discriminant Analysis (PLS-DA). Cross-validation tests were performed to estimate classification error rates ( $M$-fold method) and their statistical significance (permutation tests performed with 999 permutations) for both floral colour and odour. Variation in fruit set among subspecies and populations of the same subspecies was investigated using Wilcoxon-Mann-Whitney tests.

\subsection{2 | Estimation of selection gradients}

Phenotypic selection exerted on floral traits was quantified using the method of Lande and Arnold (1983). As a preliminary analysis, selection differentials $S$ were estimated in each population as the covariance between relative female reproductive success and standardized trait values using univariate regressions. $p$-Values were corrected using the false discovery rate (FDR) method (Verhoeven, Simonsen, \& Mclntyre, 2005). Afterwards, directional selection gradients $\beta$ were estimated in each population using multivariate regressions with relative female reproductive success as the response variable and standardized trait values as explanatory variables. Because plant size traits were highly correlated (see Table S1), only plant height was included in these regressions. Regarding floral colour traits, a global principal component analysis (PCA) was performed on the entire dataset (i.e., including all seven populations) in order to reduce the number of traits included in our models and transform potentially correlated variables into uncorrelated principal components (PCs). Scores on the first PC (accounting for $55 \%$ of the total variance) were included as an explanatory variable in our models. Regarding floral odour traits, because we had no a priori knowledge about the biological relevance of individual compounds, we chose to estimate selection exerted on entire blends of floral scents (see Method S5). To do so, as for floral colour traits, we used PCA, but this time one PCA was performed in each population. We proceeded this way because many compounds were detected in one or a few populations only (see Results), and thus including scores on global PCs in our models would amount to estimating selection on traits that may not be expressed in a given population. The correlation between PCs and individual compounds is shown in Table S5. Our final models included plant height, scores on the first colour PC, total emission rate and scores on the first two to four odour PCs (i.e., PCs accounting for at least $50 \%$ of the total variance in floral scents within each population), except in Le-Cannetdes-Maures, in which we did not have floral colour data and only included plant height and floral odour variables. Only linear terms were included in these regressions, as preliminary analyses showed no evidence of stabilizing or correlational selection. Variance inflation factors (VIFs) were computed to test for multicollinearity between explanatory variables, but they almost never exceeded 2 (i.e., they exceeded 2 for two variables of 38 , and remained below 2.1 in both cases), indicating no problem of multicollinearity (Quinn, Gerald, \& Keough, 2002).

All statistical analyses were performed using the software $\mathrm{R}$ version 3.4.3 (R Core Team, 2017).

\section{3 | RESULTS}

\subsection{Variation in floral traits and female reproductive success}

Variation in plant height, inflorescence length and number of flowers was significant when comparing populations of the same subspecies, but not when comparing subspecies (Figure 2 and Table S2).

By contrast, variation in floral colour was significant when comparing either subspecies (classification error rate $=24.7 \%, p=.001$; Figure 3 ) or populations of the same subspecies (classification error rate $=35.50 \%$ for $A$. c. coriophora and $6.69 \%$ for $A$. c. fragrans, $p=.001$ in both cases). Specifically, flowers of A. c. martrinii were dark (low mean values in the $R, G$ and $B$ channels) relative to those of A. c. fragrans (high mean values in the R, $G$ and $B$ channels), whereas flowers of $A$. c. coriophora were characterized by intermediate mean values.

Forty-eight compounds (fatty acid derivatives, benzenoids and terpenoids) were detected in the blends of A. c. coriophora, A. c. fragrans and A. c. martrinii (see Table S3), including 21 that were shared among all three taxa (Figure 4). Floral scents of A. c. coriophora, A. c. fragrans and A. c. martrinii were composed of 35,31 and 32 floral volatiles, respectively. Floral odour was well differentiated among subspecies (classification error rate $=0 \%, p=.001$; Figure 4). This differentiation was both qualitative (i.e., presence/absence of compounds) and quantitative (i.e., absolute and relative amounts of compounds). Indeed, 19 of 48 floral volatiles were taxon-specific, including some predominant compounds (Figure 4). Floral scents of all three subspecies were dominated by 1,4-dimethoxybenzene (on average $44.45 \%$ of the blend: $36.56 \%$ for $A$. c. coriophora, $44.54 \%$ for A. c. fragrans and $66.68 \%$ for A. c. martrinii). However, the second predominant compound was different in all three subspecies: flowers of A. c. coriophora emitted high amounts of 1,2,4-trimethoxybenzene (11.44\% of the blend), flowers of $A$. c. fragrans high amounts of $p$-anisaldehyde (30.05\% of the blend) and flowers of A. c. martrinii high amounts of methyl-p-anisate $(11.33 \%$ of the blend). Note that floral odours of A. c. coriophora and A. c. martrinii were more similar to each other (28 compounds in common) than to those of A. c. fragrans (22 and 21 compounds in common, respectively; Figure 4). Floral scents were also well differentiated among populations of either A. c. coriophora or A. c. fragrans (classification error rate $=0 \%$ and $p=.001$ in both cases; see Figure S2). Here again, 

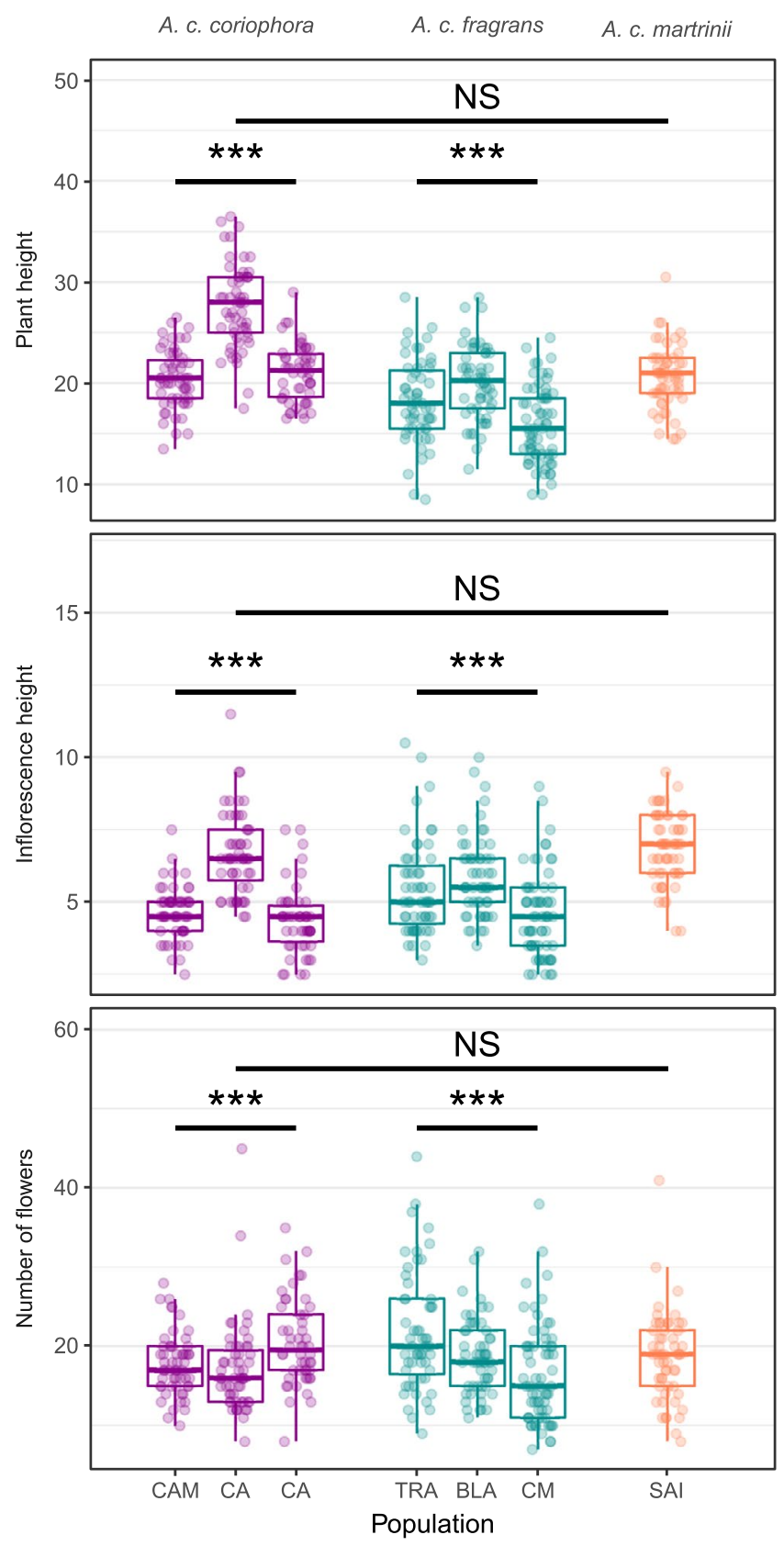

FIGURE 2 Variation in plant size traits among subspecies and populations of the same subspecies. BLA, Blandas; CA, Compssur-Artuby; CAM, Camprieu; CM, Le-Cannet-des-Maures; SAI, Saillagouse; SOU, Sournia; TRA, Trassanel; ${ }^{* * *} p<.001$; NS: nonsignificant. See Table S2 for details

this differentiation was both qualitative and quantitative, with several compounds being only found in one or two populations of three (see Table S3). Interestingly, floral odour variation within subspecies appeared to be geographically structured, with populations ordered along a longitudinal gradient on axis 2 (i.e., westernmost populations at the bottom and easternmost populations at the top of the scatterplot; see Figure S2).

Mean fruit set of open-pollinated plants varied from $30.68 \%$ in Comps-sur-Artuby to $82.84 \%$ in Trassanel, with significant variation among populations of the same subspecies $\left(F_{2,157}=33.54, p=2 \mathrm{e}^{-13}\right.$ for A. c. coriophora and $F_{2,159}=33.71, p=6 \mathrm{e}^{-13}$ for $A$. c. fragrans), but not among subspecies $\left(X^{2}(2, N=376)=0.38, p=.83\right)$.

\subsection{Estimation of selection gradients}

After correction, significant selection differentials were found for plant height, inflorescence length and number of flowers in most populations (see Table S4). By contrast, no selection was detected on floral colour traits, using either RGB values or honeybee photoreceptor excitation values (see Table S4). Regarding floral scent traits, no selection was found on total emission rate, whereas significant selection differentials were detected for absolute amounts of some compounds in two A. c. coriophora and one A. c. fragrans populations (see Table S4).

Multivariate regressions showed positive selection on plant height in all six populations of $A$. c. coriophora and A. c. fragrans, as well as positive or negative selection on at least one of the floral scent PCs in all three populations of $A$. c. fragrans (Table 1). More specifically, in Le-Cannet-des-Maures, we detected positive selection on the first PC and negative selection on the second PC (Table 1). PC1 was positively correlated with several taxon-specific compounds (namely p-anisaldehyde, 2-(4-methoxyphenyl)-ethan-1-ol, methyl-p-anisate, (E)-methylcinnamate and 3-acetylcyclohexylacetate), indicating positive selection on these volatiles. PC2 was negatively correlated with 5 -methyl- $\delta$-valerolactone and 4,5-dimethyl- $\delta$-valerolactone-here again a taxon-specific compound-indicating positive selection on these volatiles. In Blandas, we detected negative selection on the first PC (Table 1). This PC was negatively correlated with the same set of taxon-specific compounds (i.e., $p$-anisaldehyde, 2-(4-methoxyphenyl)-ethan-1-ol, methyl-p-anisate, (E)-methylcinnamate and 3-acetylcyclohexylacetate), indicating positive selection on these volatiles here again. Note that this PC was also negatively correlated with 1,2,3,5-tetramethoxybenzene, a compound that in A. c. fragrans was only found in the Blandas population. In Trassanel, we detected negative selection on the second PC (Table 1). This PC was negatively correlated with methylisohexanoate-a compound specific to the Blandas and Trassanel populations-as well as with 6-methylhept-5-en-2-one and $p$-methylanisole-two compounds that were also found in A. c. coriophora and A. c. martrinii. In A. c. coriophora, we detected no selection on floral odour in Comps-sur-Artuby, Camprieu or Sournia (Table 1). In A. c. martrinii, we found no selection on plant height nor on floral scent PCs (Table 1). No selection was found on floral colour PCs nor on total emission rate in A. c. coriophora, A. c. fragrans or A. c. martrinii (Table 1).

\section{DISCUSSION}

Phenotypic differentiation at biologically relevant traits among conspecific populations is often believed to result from divergent selective pressures imposed upon these traits. In this study, we characterized floral trait differentiation among seven populations of the widespread, generalist orchid species Anacamptis coriophora and we explored the relationship between these traits and female 


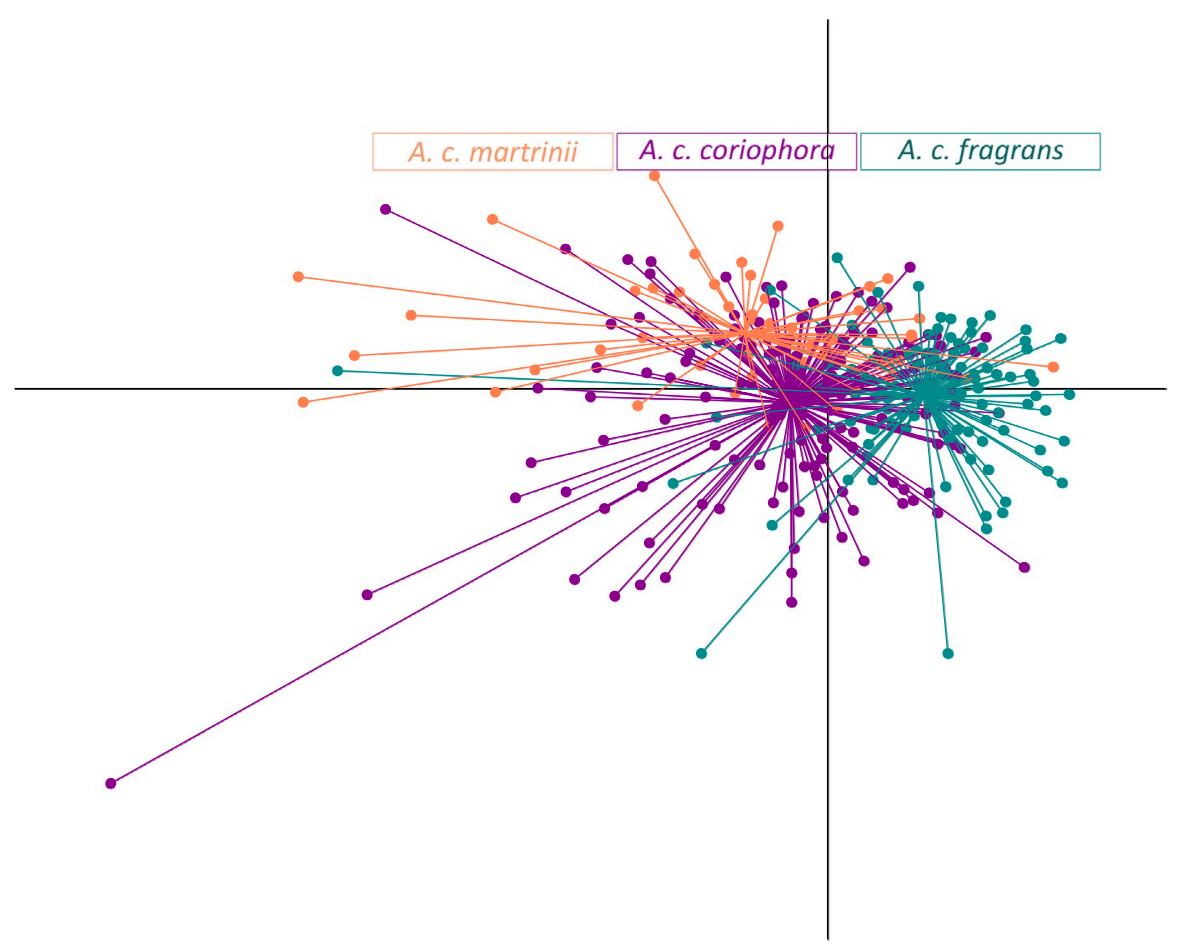

FIGURE 3 Scatter plot of the Partial Least Square-Discriminant Analysis (PLS-DA) performed on floral colour traits for the three subspecies (Anacamptis coriophora subsp. coriophora in purple, $A$. coriphora subsp. fragrans in turquoise and A. coriophora subsp. martrinii in orange)

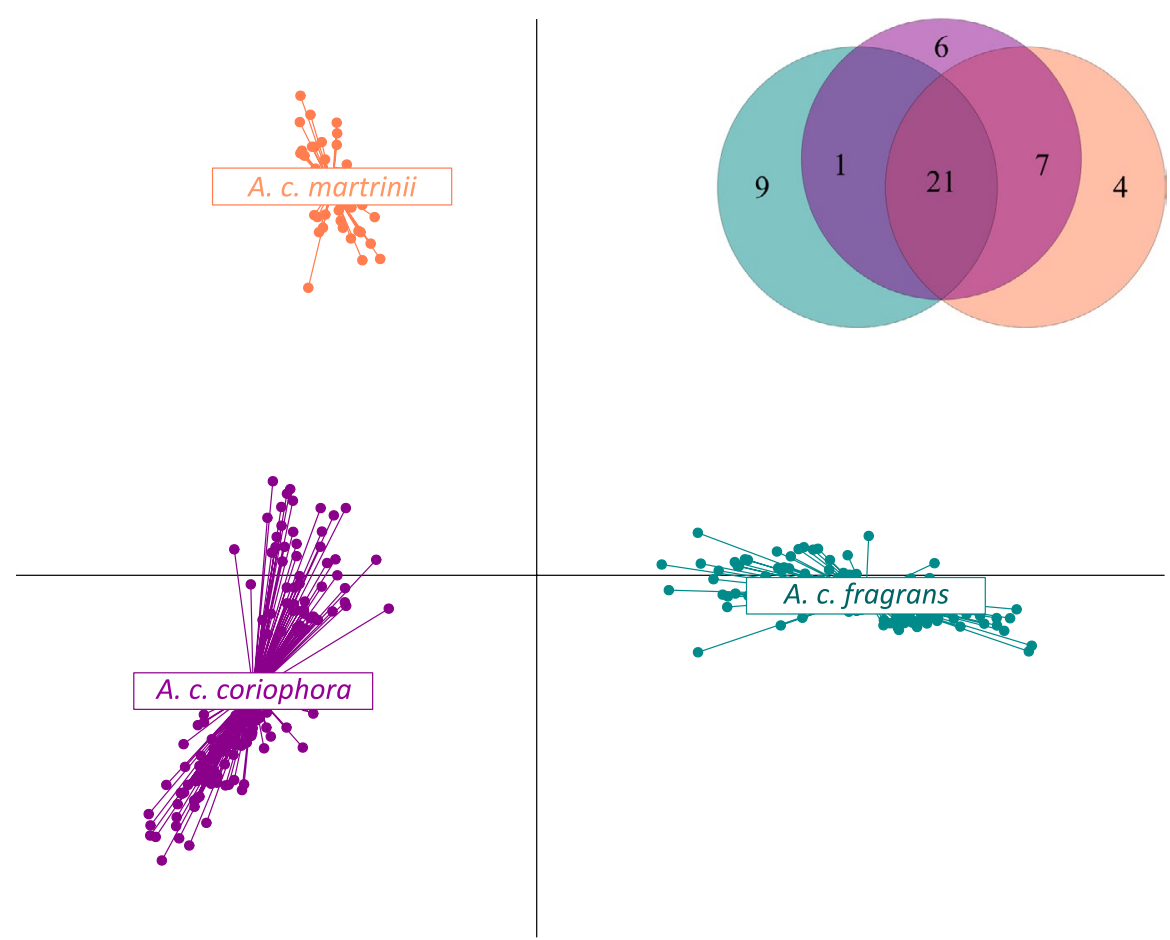

FIGURE 4 Scatter plot of the Partial Least Square-Discriminant Analysis (PLS-DA) performed on floral scent traits for the three subspecies (Anacamptis coriophora subsp. coriophora in purple, $A$. coriphora subsp. fragrans in turquoise and A. coriophora subsp. martrinii in orange), and Venn diagram showing number of taxon-specific compounds

reproductive success. We found a significant differentiation both among and within subspecies in terms of floral colour and scents, with one of the two predominant compounds emitted by each subspecies being taxon-specific. We hypothesized that such variation in floral colour and odour was mirrored by variation in phenotypic selection exerted on these traits. In particular, one of our expectations was to detect positive selection on taxon-specific floral volatiles. We indeed found such a pattern in A. c. fragrans, but not in A. c. coriophora and A. c. martrinii. This partial match between the observed pattern of chemical differentiation and phenotypic selection exerted on floral scents suggests that selective processes could contribute to ongoing floral odour divergence among A. coriophora subspecies. On the other hand, we observed no such trend for floral colour, suggesting that other processes may govern floral colour evolution in A. coriophora.

The three studied subspecies were characterized by comparable plant height, inflorescence length and number of flowers, with variation among populations of the same subspecies. We found a positive correlation between plant height and female reproductive success in most populations. Such a correlation has been documented in other plants (Gervasi \& Schiestl, 2017; Gómez, 2007), including 
TAB LE 1 Selection gradients $\beta \pm S E$ on plant height, total emission rate and principal components (PCs) of the PCAs performed on floral colour and odour data. In each population, selection gradients are extracted from one multiple regression including all floral traits (i.e., one column corresponds to one model). Odour PCs are population-specific (i.e., one individual PCA performed in each population)

\begin{tabular}{|c|c|c|c|c|c|c|c|}
\hline$\frac{\text { Subspecies }}{\text { Population }}$ & \multicolumn{3}{|l|}{ COR } & \multicolumn{3}{|l|}{ FRA } & $\frac{\text { MAR }}{\text { SAI }}$ \\
\hline Plant height & $0.14 \pm 0.04^{* *}$ & $0.43 \pm 0.13^{* *}$ & $0.12 \pm 0.05^{*}$ & $0.22 \pm 0.06^{* * *}$ & $0.37 \pm 0.13^{* *}$ & $0.28 \pm 0.06^{* * *}$ & $0.03 \pm 0.08$ \\
\hline Colour PC1 & $0.002 \pm 0.04$ & $0.08 \pm 0.12$ & $-0.02 \pm 0.05$ & $-0.02 \pm 0.06$ & - & $0.02 \pm 0.05$ & $-0.04 \pm 0.08$ \\
\hline Total emission rate & $0.05 \pm 0.05$ & $-0.20 \pm 0.17$ & $-0.03 \pm 0.07$ & $-0.08 \pm 0.08$ & $0.10 \pm 0.14$ & $0.08 \pm 0.06$ & $-0.02 \pm 0.12$ \\
\hline Odour PC1 & $-0.02 \pm 0.05$ & $-0.16 \pm 0.17$ & $-0.09 \pm 0.06$ & $-0.17 \pm 0.07^{*}$ & $0.27 \pm 0.12^{*}$ & $-0.08 \pm 0.06$ & $0.02 \pm 0.11$ \\
\hline Odour PC3 & $-0.02 \pm 0.04$ & - & - & - & - & $0.08 \pm 0.05$ & $0.02 \pm 0.08$ \\
\hline Odour PC4 & $-0.04 \pm 0.04$ & - & - & - & - & - & - \\
\hline
\end{tabular}

Note: The number of PCs included in each model was chosen to explain at least $50 \%$ of the total variance in floral scents within each population. Abbreviations: BLA, Blandas; CA, Comps-sur-Artuby; CAM, Camprieu; CM, Le-Cannet-des-Maures; COR, Anacamptis coriophora coriophora; FRA, A. c. fragrans; MAR, A. coriophora c. martrinii; SAI: Saillagouse; SOU, Sournia; TRA: Trassanel.

${ }^{*} p<.05 ;{ }^{* *} p<.01 ;{ }^{* * *} p<.001$.

orchids (Sletvold et al., 2010; Sletvold, Trunschke, Wimmergren, \& Ägren, 2012), and could be mediated by pollinators, as taller individuals may be more easily detected by foraging insects (Donnelly, Lortie, \& Aarssen, 1998). However, because plant height was correlated to number of flower in most populations, its apparent effect on female reproductive success may be due to the obvious correlation between flower and fruit numbers. In addition, plant height may be linked to other floral or vegetative genetically coded traits affecting female reproductive success, causing indirect selection on this character (Alexandersson \& Johnson, 2002; Gómez, 2000). Alternatively, nongenetic factors such as plant age or microhabitat could affect plant height and fruit set in a parallel fashion and explain this correlation.

The three studied subspecies were characterized by different floral colours, yet no phenotypic selection was found on this trait, contrary to what has been reported in other orchids (Gross et al., 2016; Sletvold, Trunschke, Smit, Verbeek, \& Ågren, 2016). Both visual and olfactory signals are known to be important for bee attraction (Burger et al., 2010), but visual traits may be of secondary importance compared to olfactory traits for $A$. coriophora pollination. Alternatively, this finding could be explained by conflicting selective pressures imposed by mutualists and antagonists (Frey, 2004). The fact that we did not detect any selection on floral colour despite significant differentiation both among and within subspecies could either suggest that selection has driven floral colour divergence in the past but is now relaxed (or too weak to be detected with our limited sample size), or that other processes, such as genetic drift, are responsible for the observed pattern of floral colour differentiation.

The three studied subspecies were also characterized by different floral scents, with two predominant compounds per subspecies, one shared among all three taxa (1,4-dimethoxybenzene) and one taxon-specific (1,2,4-trimethoxybenzene in A. c. coriophora, $p$-anisaldehyde in A. c. fragrans and methyl-p-anisate in A. c. martrinii). In addition, many other taxon-specific floral volatiles were found in the blends of the three studied subspecies, including fatty acid derivatives, benzenoids and terpenoids. Our results are congruent with previous reports on the floral odour chemistry of A. c. fragrans (Dormont, Delle-Vedove, Bessière, \& Schatz, 2014; Salzmann, Nardella, Cozzolino, \& Schiestl, 2007), whereas floral scents of A. c. coriophora and A. c. martrinii were analysed for the first time in this study. In particular, we demonstrated that A. c. martrinii emits significant amounts of floral volatiles, despite being described as odourless in the literature (Delforge, 2006). Because the three studied subspecies grow in different habitats, probably characterized by distinct microclimatic conditions and biotic communities, phenotypic plasticity cannot be ruled out as a potential driver of floral odour variation in A. coriophora (Majetic, Raguso, \& Ashman, 2009). In fact, there is growing evidence that plants can plastically respond to increasing temperatures (Farré-Armengol, 2014), drought (Campbell, Sosenski, \& Raguso, 2019) or other biotic or abiotic factors (Schiestl, Kirk, Bigler, Cozzolino \& Desurmont, 2014) through quantitative changes in floral scent emissions (i.e., changes in total emission rate or in the relative amounts of the different compounds). A common garden experiment would have allowed us to disentangle the relative importance of environmental versus genetic factors for floral odour variation, but such experiments are difficult to set up in slow-growing plants such as orchids. However, it seems unlikely that the consistent difference in the predominant floral volatiles emitted by the three studied subspecies is due to phenotypic plasticity alone, and it seems reasonable to assume that such difference is at least partly genetically based. If this assumption holds, floral scent differentiation among subspecies may be the outcome of selective and/or neutral processes. Compared to other floral traits, few phenotypic selection studies have dealt with scents so far, despite the well-known implication of olfactory signals in biotic interactions, particularly pollination (Raguso, 2008). Evidence from these studies suggests that selection usually favours the most odorous plants (Majetic et al., 2009; Parachnowitsch, Raguso, \& Kessler, 2012), or plants emitting the highest amounts of a few biologically relevant compounds (Chapurlat, Ågren, Anderson, Friberg, \& Sletvold, 2019; Parachnowitsch et al., 2012; Schiestl, Huber, \& Gomez, 2011). Here, we found no significant selection gradients on total emission rate, nor did we find consistently significant selection differentials on amounts 
of specific floral volatiles. By contrast, when considering entire blends of floral scents, we detected phenotypic selection on sets of correlated compounds-mostly taxon-specific floral volatiles-in all three populations of A. c. fragrans. Although other selective agents could account for this, pollinators may play a role here. First, several of the compounds found to be under positive selection are known to be involved in the chemical communication between plants and pollinators. For example, the benzenoid $p$-anisaldehyde is known to constitute an honest signal of reward for pollinating bees (Knauer \& Schiestl, 2015) and was shown to be under pollinator-mediated selection in Brassica rapa plants (Gervasi \& Schiestl, 2017). Second, fruit set was significantly (although not strongly, as expected in this rewarding species) pollen-limited in all three populations of $A$. c. fragrans (see Figure S3), indicating that pollinators may act as selective agents in these populations (Sletvold \& Ågren, 2014; Trunschke, Sletvold, \& Ågren, 2017). On the other hand, we detected no phenotypic selection on floral scents of $A$. c. coriophora and A. c. martrinii, despite the fact that female reproductive success was significantly pollen-limited at least in A. c. martrinii (see Figure S3). This finding could be explained by conflicting selective pressures imposed by mutualists and antagonists (Schiest et al., 2011), especially since some compounds emitted by these two subspecies-including 1,2,4-trimethoxybenzene-are known to attract both pollinators and herbivores (Andrews, Theis, \& Adler, 2007). Alternatively, floral scents may not play a predominant role in A. c. coriophora and A. c. martrinii pollination. The ecological function of floral odour in A. coriophora and its link with other fitness components, particularly pollen export, should be studied more thoroughly to better understand the selective pressures at work here.

In the orchid Gymnadenia odoratissima, selection on floral colour and scents was shown to differ between lowland and mountain populations and was broadly congruent with the observed pattern of floral trait differentiation between these populations (Gross et al., 2016). Likewise, we showed a partial match between the observed pattern of chemical differentiation and selective pressures imposed upon floral odour in A. coriophora. This suggests that selective processes could contribute to ongoing floral scent divergence among A. coriophora subspecies, potentially along with other processes. Population genetic studies are needed to assess whether selective and neutral processes act in concert to drive such divergence, or whether selection promotes phenotypic differentiation despite substantial gene flow among subspecies within geographical regions. In any case, it should be pointed out that such a scenario relies on two important assumptions: first, that phenotypic selection is consistent across years, which was not tested here; and second, that variation in floral scents is heritable. However, floral size or shape traits often exhibit relatively high heritabilities (Ashman \& Majetic, 2006), little is known regarding the heritability of floral odour, although macroevolutionary studies showing consistent associations between functional groups of pollinators and blends of floral scents suggest that the latter are evolvable traits (Harder \& Johnson, 2009). Accordingly, an artificial selection experiment on Brassica rapa has shown that floral odour can evolve rapidly under phenotypic selection (Zu, Blanckenhorn, \& Schiestl, 2016). However, whether this is the case in our study system remains to be tested.
It is worth noting that contrary to Gross et al. (2016), who showed consistent differences in the pollinator guilds associated with lowland versus mountain populations of $G$. odoratissima, we did not find any obvious associations between A. coriophora subspecies and taxonomic or functional groups of pollinators, at least for A. c. coriophora and A. c. fragrans (see Table S6). In fact, our data suggest that pollinator communities vary among all seven sites, regardless of the A. coriophora subspecies growing locally. More data on the composition of pollinator communities and on the contribution of the various pollinator species to reproductive success are needed to understand the role potentially played by pollinators in the diversification of $A$. coriophora.

To our knowledge, this study is the first to document variation in phenotypic selection exerted on floral scents among subspecies. Our results suggest that selection could contribute to ongoing chemical differentiation between $A$. coriophora $c$. fragrans on the one hand and A. c. coriophora and A. c. martrinii on the other hand. The contribution of selection to the marked phenotypic differentiation evidenced among $A$. coriophora subspecies-relative to that of neutral processes-remains to be assessed, by analysing patterns of genetic differentiation among the latter. Further studies are also needed to assess whether such differentiation represents the early stages of speciation, by testing whether floral traits can promote prezygotic reproductive isolation among these subspecies.

\section{ACKNOWLEDGMENTS}

This work was supported by HESAM Université and its Paris Nouveaux Mondes programme, the ARSENIC programme [ANR14-CE02-0012: "Adaptation and Resilience of Spatial Ecological Networks to human-Induced Changes »] and the BIODIVMEX programme (Biodiversity of the Mediterranean experiment, CNRS). The Direction Régionale de l'Environnement, de I'Aménagement et du Logement (DREAL) Occitanie provided sampling permits (no. 2016-s-14). We thank the Plateforme d'Analyses Chimiques en Ecologie (PACE) of the Labex CEMEB for technical assistance. We are grateful to the Société Française d'Orchidophilie (SFO) and to M. Audax, F. Barreda, R. Buscail, M. Grenié, A. Grimaud, L. Valois and D. Vizcaino for their help in the field. We thank J. Troscianko for measuring the receptor sensitivity of our camera, D. Genoud for identifying pollinators and D. McKey (University of Montpellier), N. Sletvold and P. Milesi (University of Uppsala) for helpful discussions. The data that support the findings of this study are available in Dryad (https://doi.org/10.5061/dryad.kprr4xh29).

\section{ORCID}

Nina Joffard iD https://orcid.org/0000-0003-3712-6080

\section{REFERENCES}

Adams, R. (2007). Identification of essential oil components by gas chromatography/mass spectrometry. Carol Stream, IL: Allured Publishing Corporation.

Alexandersson, R., \& Johnson, S. D. (2002). Pollinator-mediated selection on flower-tube length in a hawkmoth-pollinated Gladiolus (Iridaceae). Proceedings of the Royal Society B: Biological Sciences, 269(1491), 631636. https://doi.org/10.1098/rspb.2001.1928 
Anderson, B., Ros, P., Wiese, T. J., \& Ellis, A. G. (2014). Intraspecific divergence and convergence of floral tube length in specialized pollination interactions. Proceedings of the Royal Society B: Biological Sciences, 281(1795), 20141420. https://doi.org/10.1098/rspb.2014.1420

Andrews, E. S., Theis, N., \& Adler, L. S. (2007). Pollinator and herbivore attraction to Cucurbita floral volatiles. Journal of Chemical Ecology, 33(9), 1682-1691. https://doi.org/10.1007/s10886-007-9337-7

Arista, M., Talavera, M., Berjano, R., \& Ortiz, P. L. (2013). Abiotic factors may explain the geographical distribution of flower colour morphs and the maintenance of colour polymorphism in the scarlet pimpernel. Journal of Ecology, 101(6), 1613-1622. https://doi. org/10.1111/1365-2745.12151

Armbruster, W. S. (2002). Can indirect selection and genetic context contribute to trait diversification? A transition-probability study of blossom-colour evolution in two genera. Journal of Evolutionary Biology, 15(3), 468-486. https://doi.org/10.1046/j.1420-9101.2002.00399.x

Ashman, T. L., \& Majetic, C. J. (2006). Genetic constraints on floral evolution: A review and evaluation of patterns. Heredity, 96(5), 343. https://doi.org/10.1038/sj.hdy.6800815

Bateman, R., Hollingsworth, P., Preston, J. C., Yi-Bo, L., Pridgeon, A. Chase, M., \& Hollingsworth, M. (2003). Molecular phylogenetics and evolution of Orchidinae and selected Habenarinae (Orchidaceae). Botanical Journal of the Linnean Society, 142(1), 1-40. https://doi. org/10.1046/j.1095-8339.2003.00157.x

Bateman, R. M., Pridgeon, A. M. \& Chase, M. W.. (2005). Phylogenetics of subtribe Orchidinae (Orchidoideae, Orchidaceae) based on nuclear ITS sequences. Lindleyana, 20, 120.

Bournérias, M., Prat, D. \& the Société Française d'Orchidophilie. (2005). Les Orchidées de France. Belgique et Luxembourg, deuxième édition. Mèze, France: Biotope(collection Parthénope).

Breitkopf, H., Schlüter, P. M., Xu, S., Schiestl, F. P., Cozzolino, S., \& Scopece, G. (2013). Pollinator shifts between Ophrys sphegodes populations: Might adaptation to different pollinators drive population divergence? Journal of Evolutionary Biology, 26(10), 2197-2208. https://doi.org/10.1111/jeb.12216

Burger, H., Dötterl, S., \& Ayasse, M. (2010). Host-plant finding and recognition by visual and olfactory floral cues in an oligolectic bee. Functional Ecology, 24(6), 1234-1240. https://doi.org/10.1111/j.1365-2435.2010.01744.x

Campbell, P., Sosenski, S., \& Raguso, R. A. (2019). Phenotypic plasticity of floral volatiles in response to increasing drought stress. Annals of botany, 123, 601-610.

Caruso, C. M. (2000). Competition for pollination influences selection on floral traits of Ipomopsis aggregata. Evolution, 54(5), 1546-1557. https://doi.org/10.1111/j.0014-3820.2000.tb00700.x

Caruso, C. M. (2001). Differential selection on floral traits of Ipomopsis aggregata growing in contrasting environments. Oikos, 94(2), 295302. https://doi.org/10.1034/j.1600-0706.2001.940210.x

Caruso, C. M., Eisen, K. E., Martin, R. A., \& Sletvold, N. (2019). A meta-analysis of the agents of selection on floral traits. Evolution, 73(1), 41-44. https://doi.org/10.1111/evo.13639

Chapurlat, E., Ågren, J., Anderson, J., Friberg, M., \& Sletvold, N. (2019). Conflicting selection on floral scent emission in the orchid Gymnadenia conopsea. New Phytologist, 222(4), 2009-2022. https:// doi.org/10.1111/nph.15747

Chapurlat, E., Ågren, J., \& Sletvold, N. (2015). Spatial variation in pollinator-mediated selection on phenology, floral display and spur length in the orchid Gymnadenia conopsea. New Phytologist, 208(4), 12641275. https://doi.org/10.1111/nph.13555

Chess, S. K., Raguso, R. A., \& LeBuhn, G. (2008). Geographic divergence in floral morphology and scent in Linanthus dichotomus (Polemoniaceae). American Journal of Botany, 95(12), 1652-1659. https://doi.org/10.3732/ajb.0800118

Claessens, J., \& Kleynen, J. (2011). The flower of the European orchid: Form and function. Geulle, The Netherlands: Jean Claessens and Jacques Kleynen.
Dafni, A., \& Ivri, Y. (1979). Pollination ecology of, and hybridization between, Orchis coriophora L. and O. collina Sol. Ex Russ. in Israel. New Phytologist, 83, 181-187. https://doi.org/10.1111/j.1469-8137.1979. tb00740.x

Darwin, C. R. (1862). On the various contrivances by which British and foreign orchids are fertilised by insects and on the good effect of intercrossing. London, UK: Murray.

Delforge, P. (2006). Orchids of Europe, North Africa and the Middle East. London, UK: A. and C. Black.

Delle-Vedove, R., Schatz, B., \& Dufay, M. (2017). Understanding intraspecific variation of floral scent in light of evolutionary ecology. Annals of Botany, 120(1), 1-20. https://doi.org/10.1093/aob/mcx055

Donnelly, S. E., Lortie, C. J., \& Aarssen, L. W. (1998). Pollination in Verbascum thapsus (Scrophulariaceae): The advantage of being tall. American Journal of Botany, 85(11), 1618-1625. https://doi. org $/ 10.2307 / 2446490$

Dormont, L., Delle-Vedove, R., Bessière, J. M., \& Schatz, B. (2014). Floral scent emitted by white and coloured morphs in orchids. Phytochemistry, 100, 51-59. https://doi.org/10.1016/j.phyto chem.2014.01.009

Dormont, L., Joffard, N., \& Schatz, B. (2019). Intraspecific variation in floral color and odor in orchids. International Journal of Plant Sciences, 180(9), 1036-1058. https://doi.org/10.1086/705589

Ellis, A. G., \& Johnson, S. D. (2009). The evolution of floral variation without pollinator shifts in Gorteria diffusa (Asteraceae). American Journal of Botany, 96(4), 793-801. https://doi.org/10.3732/ajb.0800222

Farré-Armengol, G., Filella, I., Llusià, J., Niinemets, Ü, \& Peñuelas, J. (2014). Changes in floral bouquets from compound-specific responses to increasing temperatures. Global change biology, 20, 3660-3669.

Frey, F. M. (2004). Opposing natural selection from herbivores and pathogens may maintain floral-color variation in Claytonia virginica (Portulacaceae). Evolution, 58(11), 2426-2437. https://doi. org/10.1111/j.0014-3820.2004.tb00872.x

Gervasi, D. D. L., \& Schiestl, F. P. (2017). Real-time divergent evolution in plants driven by pollinators. Nature Communications, 8, 14691. https://doi.org/10.1038/ncomms14691

Gómez, J. M. (2000). Phenotypic selection and response to selection in Lobularia maritima: Importance of direct and correlational components of natural selection. Journal of Evolutionary Biology, 13(4), 689-699. https://doi.org/10.1046/j.1420-9101.2000.00196.x

Gómez, J. M. (2007). Dispersal-mediated selection on plant height in an autochorously dispersed herb. Plant Systematics and Evolution, 268(1-4), 119-130. https://doi.org/10.1007/s00606-007-0568-4

Gómez, J. M., Bosch, J., Perfectti, F., Fernández, J. D., Abdelaziz, M., \& Camacho, J. P. M. (2008). Spatial variation in selection on corolla shape in a generalist plant is promoted by the preference patterns of its local pollinators. Proceedings of the Royal Society B: Biological Sciences, 275(1648), 2241-2249. https://doi.org/10.1098/ rspb.2008.0512

Gómez, J. M., Perfectti, F., Bosch, J., \& Camacho, J. P. M. (2009). A geographic selection mosaic in a generalized plant-pollinator-herbivore system. Ecological Monographs, 79(2), 245-263. https://doi. org/10.1890/08-0511.1

Gross, K., Sun, M., \& Schiestl, F. P. (2016). Why do floral perfumes become different? Region-specific selection on floral scent in a terrestrial orchid. PLoS One, 11(2), e0147975. https://doi.org/10.1371/ journal.pone. 0147975

Hall, M. C., \& Willis, J. H. (2006). Divergent selection on flowering time contributes to local adaptation in Mimulus guttatus populations. Evolution, 60(12), 2466-2477. https://doi.org/10.1111/j.0014-3820.2006. tb01882.x

Harder, L. D., \& Barrett, S. C. H. (2006). Ecology and evolution of flowers. Oxford, UK: Oxford University Press.

Harder, L. D., \& Johnson, S. D. (2009). Darwin's beautiful contrivances: Evolutionary and functional evidence for 
floral adaptation. New Phytologist, 183(3), 530-545. https://doi. org/10.1111/j.1469-8137.2009.02914.x

Irwin, R. E., Bronstein, J. L., Manson, J. S., \& Richardson, L. (2010). Nectar robbing: Ecological and evolutionary perspectives. Annual Review of Ecology, Evolution, and Systematics, 41(1), 271-292. https://doi. org/10.1146/annurev.ecolsys.110308.120330

Jersáková, J., Jürgens, A., Šmilauer, P., \& Johnson, S. D. (2012). The evolution of floral mimicry: Identifying traits that visually attract pollinators. Functional Ecology, 26(6), 1381-1389. https://doi. org/10.1111/j.1365-2435.2012.02059.x

Joffard, N., Massol, F., Grenié, M., Montgelard, C., \& Schatz, B. (2019). Effect of pollination strategy, phylogeny and distribution on pollination niches of Euro-Mediterranean orchids. Journal of Ecology, 107(1), 478-490. https://doi.org/10.1111/1365-2745.13013

Johnson, S. D., \& Steiner, K. E. (1997). Long-tongued fly pollination and evolution of floral spur length in the Disa draconis complex (Orchidaceae). Evolution, 51(1), 45-53. https://doi. org/10.2307/2410959

Knauer, A. C., \& Schiestl, F. P. (2015). Bees use honest floral signals as indicators of reward when visiting flowers. Ecology Letters, 18(2), 135-143. https://doi.org/10.1111/ele.12386

Lande, R., \& Arnold, S. (1983). The measurement of selection on correlated characters. Evolution, 37(6), 1210-1226. https://doi. org $/ 10.2307 / 2408842$

Majetic, C. J., Raguso, R. A., \& Ashman, T. L. (2009). The sweet smell of success: Floral scent affects pollinator attraction and seed fitness in Hesperis matronalis. Functional Ecology, 23(3), 480-487. https://doi. org/10.1111/j.1365-2435.2008.01517.x

McCall, A. C., Murphy, S. J., Venner, C., \& Brown, M. (2013). Florivores prefer white versus pink petal color morphs in wild radish, Raphanus sativus. Oecologia, 172(1), 189-195. https://doi.org/10.1007/s0044 2-012-2480-z

Parachnowitsch, A. L., \& Caruso, C. M. (2008). Predispersal seed herbivores, not pollinators, exert selection on floral traits via female fitness. Ecology, 89(7), 1802-1810. https://doi.org/10.1890/07-0555.1

Parachnowitsch, A. L., Raguso, R. A., \& Kessler, A. (2012). Phenotypic selection to increase floral scent emission, but not flower size or colour in bee-pollinated Penstemon digitalis. New Phytologist, 195(3), 667-675. https://doi.org/10.1111/j.1469-8137.2012.04188.x

Quinn, G. P., Gerald, P., \& Keough, M. J. (2002). Experimental design and data analysis for biologists. Cambridge, UK: Cambridge University Press.

R Core Team (2017). R: A language and environment for statistical computing. Vienna, Austria: R Foundation for Statistical Computing. Retrieved from http://www.R-project.org

Raguso, R. A. (2008). Wake up and smell the roses: The ecology and evolution of floral scent. Annual Review of Ecology, Evolution, and Systematics, 39, 549-569. https://doi.org/10.1146/annurev.ecolsys.38.091206.095601

Ramos, S. E., \& Schiestl, F. P. (2019). Rapid plant evolution driven by the interaction of pollination and herbivory. Science, 364(6436), 193196. https://doi.org/10.1126/science.aav6962

Salzmann, C. C., Nardella, A. M., Cozzolino, S., \& Schiestl, F. P. (2007). Variability in floral scent in rewarding and deceptive orchids: The signature of pollinator-imposed selection? Annals of Botany, 100(4), 757-765. https://doi.org/10.1093/aob/mcm161

Sandring, S., Riihimäki, M. A., Savolainen, O., \& Ågren, J. (2007). Selection on flowering time and floral display in an alpine and a lowland population of Arabidopsis lyrata. Journal of Evolutionary Biology, 20(2), 558-567. https://doi.org/10.1111/j.1420-9101.2006.01260.x

Schiestl, F. P., Huber, F. K., \& Gomez, J. M. (2011). Phenotypic selection on floral scent: Trade-off between attraction and deterrence? Evolutionary Ecology, 25(2), 237-248. https://doi.org/10.1007/s1068 2-010-9409-y

Schiestl, F. P., \& Johnson, S. D. (2013). Pollinator-mediated evolution of floral signals. Trends in Ecology and Evolution, 28(5), 307-315. https:// doi.org/10.1016/j.tree.2013.01.019
Schiestl, F. P., Kirk, S. H., Bigler, L., Cozzolino, S., \& Desurmont, G. A. (2014). Herbivory and floral signaling: phenotypic plasticity and tradeoffs between reproduction and indirect defense.. New Phytologist, 203, 257-266. https://doi.org/10.1016/j.tree.2013.01.019

Schneider, C. A., Rasband, W. S., \& Eliceiri, K. W. (2012). NIH Image to Image J: 25 years of image analysis. Nature Methods, 9(7), 671-675. https://doi.org/10.1038/nmeth.2089

Sletvold, N., \& Ågren, J. (2014). There is more to pollinator-mediated selection than pollen limitation. Evolution, 68(7), 1907-1918. https:// doi.org/10.1111/evo.12405

Sletvold, N., Grindeland, J. M., \& Ågren, J. (2010). Pollinator-mediated selection on floral display, spur length and flowering phenology in the deceptive orchid Dactylorhiza lapponica. New Phytologist, 188(2), 385-392. https://doi.org/10.1111/j.1469-8137.2010.03296.x

Sletvold, N., Trunschke, J., Smit, M., Verbeek, J., \& Ågren, J. (2016). Strong pollinator-mediated selection for increased flower brightness and contrast in a deceptive orchid. Evolution, 70(3), 716-724. https:// doi.org/10.1111/evo.12881

Sletvold, N., Trunschke, J., Wimmergren, C., \& Ägren, J. (2012). Separating selection by diurnal and nocturnal pollinators on floral display and spur length in Gymnadenia conopsea. Ecology, 93(8), 1880-1891. https://doi.org/10.1890/11-2044.1

Soteras, F., Rubini Pisano, M. A., Bariles, J. B., Moré, M., \& Cocucci, A. A. (2020). Phenotypic selection mosaic for flower length influenced by geographically varying hawkmoth pollinator proboscis length and abiotic environment. New Phytologist, 225(2), 985-998. https://doi. org/10.1111/nph.16192

Sprengel, C. K. (1793). Das entdeckte Geheimniss der Natur im Bau und in der Befruchtung der Blumen. Berlin, Germany: F. Vieweg.

Strauss, S. Y., \& Whittall, J. B. (2006). Non-pollinator agents of selection on floral traits. Ecology and Evolution of Flowers, 2006, 120-138.

Totland, $\varnothing$. (2001). Environment-dependent pollen limitation and selection on floral traits in an alpine species. Ecology, 82(8), 2233-2244. https://doi.org/10.2307/2680228

Troscianko, J., \& Stevens, M. (2015). Image calibration and analysis toolbox - A free software suite for objectively measuring reflectance, colour and pattern. Methods in Ecology and Evolution, 6(11), 13201331. https://doi.org/10.1111/2041-210X.12439

Trunschke, J., Sletvold, N., \& Ågren, J. (2017). Interaction intensity and pollinator-mediated selection. New Phytologist, 214(3), 1381-1389. https://doi.org/10.1111/nph.14479

Verhoeven, K. J. F., Simonsen, K. L., \& McIntyre, L. M. (2005). Implementing false discovery rate control: Increasing your power. Oikos, 108(3), 643-647. https://doi.org/10.1111/j.0030-1299.2005.13727.x

Whitehead, M. R., \& Peakall, R. (2009). Integrating floral scent, pollination ecology and population genetics. Functional Ecology, 23(5), 863874. https://doi.org/10.1111/j.1365-2435.2009.01620.x

Zu, P., Blanckenhorn, W. U., \& Schiestl, F. P. (2016). Heritability of floral volatiles and pleiotropic responses to artificial selection in Brassica rapa. New Phytologist, 209(3), 1208-1219. https://doi.org/10.1111/ nph.13652

\section{SUPPORTING INFORMATION}

Additional supporting information may be found online in the Supporting Information section.

How to cite this article: Joffard N, Le Roncé I, Langlois A, et al. Floral trait differentiation in Anacamptis coriophora: Phenotypic selection on scents, but not on colour. J Evol Biol. 2020;33:1028-1038. https://doi.org/10.1111/jeb.13657 